Pacific

Journal of

Mathematics

\title{
CONVERGENCE TO STEADY STATES FOR A ONE-DIMENSIONAL VISCOUS HAMILTON-JACOBI EQUATION WITH DIRICHLET BOUNDARY CONDITIONS
}

PHILIPPE LAURENÇOT 


\title{
CONVERGENCE TO STEADY STATES FOR A ONE-DIMENSIONAL VISCOUS HAMILTON-JACOBI EQUATION WITH DIRICHLET BOUNDARY CONDITIONS
}

\author{
PhilipPe LAURENÇOT
}

\begin{abstract}
We investigate the convergence to steady states of the solutions to the onedimensional viscous Hamilton-Jacobi equation $\partial_{t} u-\partial_{x}^{2} u=\left|\partial_{x} u\right|^{p}$, where $(t, x) \in(0, \infty) \times(-1,1)$ and $p \in(0,1)$, with homogeneous Dirichlet boundary conditions. For that purpose, a Liapunov functional is constructed by the approach of Zelenyak (1968). Instantaneous extinction of $\partial_{x} u$ on a subinterval of $(-1,1)$ is shown for suitable initial data.
\end{abstract}

\section{Introduction}

Nonnegative solutions to the one-dimensional viscous Hamilton-Jacobi equation

$$
\begin{aligned}
\partial_{t} u-\partial_{x}^{2} u & =a\left|\partial_{x} u\right|^{p}, \quad(t, x) \in(0, \infty) \times(-1,1), \\
u(t, \pm 1) & =0, \quad t \in(0, \infty), \\
u(0) & =u_{0} \geq 0, \quad x \in(-1,1),
\end{aligned}
$$

exhibit a rich variety of qualitative behaviours, according to the sign of $a \in\{-1,1\}$ and the values of $p \in(0, \infty)$. On the one hand, extinction in finite time (that is, there is $T_{\star}>0$ such that $u(t) \equiv 0$ for $\left.t \geq T_{\star}\right)$ occurs for $a=-1$ and $p \in(0,1)$, while $u(t)$ converges exponentially fast to zero as $t \rightarrow \infty$ if $a=-1$ and $p \geq 1$ [Benachour et al. 2007]. On the other hand, if $a=1$ and $p>2$, finite time gradient blow-up takes place for suitably large initial data [Souplet 2002] while convergence to zero of $u(t)$ as $t \rightarrow \infty$ still holds true for global solutions [Arrieta et al. 2004; Souplet and Zhang 2006]. In addition, all solutions are global for $a=1$ and $p \in[1,2]$ and converge to zero as $t \rightarrow \infty$ [Benachour et al. 2007; Souplet and Zhang 2006].

The case $a=1$ and $p \in(0,1)$ offers an interesting novelty and is the subject of the present paper. Indeed, in contrast to the previous cases, the initial-boundary value problem (1)-(3) has a one parameter family $\left(U_{\vartheta}\right)_{\vartheta \in[0,1]}$ of steady states when $a=1$ and $p \in(0,1)$ with $U_{1} \equiv 0$ and $U_{\vartheta}$ is not constant if $\vartheta \in[0,1)$. These steady

MSC2000: primary 35B40; secondary 35K55, 37B25.

Keywords: diffusive Hamilton-Jacobi equation, convergence to steady states, gradient extinction,

Liapunov functional. 
states play an important role in the dynamics of solutions to (1)-(3): indeed, we will prove that any solution $u$ to (1)-(3) converges as $t \rightarrow \infty$ towards a steady state, which is nontrivial if, for instance, the initial datum $u_{0}$ is nonnegative with a positive maximum. An interesting feature of $U_{\vartheta}$ for $\vartheta \in(0,1)$ is that they are constant on a subinterval of $(-1,1)$. This property is of course related to the fact that $p$ ranges in $(0,1)$ and is reminiscent of the finite time extinction phenomenon already alluded to for nonnegative solutions when $a=-1$ and $p \in(0,1)$. It is then natural to wonder whether the nonlinear term $\left|\partial_{x} u\right|^{p}$ may induce a similar singular behaviour on the dynamics of $u$. More precisely, for a particular class of nonnegative initial data, we will show that the gradient $\partial_{x} u$ vanishes identically on $\left[T_{\star}, \infty\right) \times I$ for some $T_{\star}>0$ and some subinterval $I$ of $(-1,1)$. Let us point out here that, for nonnegative initial data, extinction in finite time cannot occur when $a=1$ and $p \in(0,1)$, for the comparison principle warrants that $u$ is bounded from below by the solution to the linear heat equation with the same initial and boundary data.

From now on, we thus assume that

$$
a=1 \text { and } p \in(0,1),
$$

and

$$
u_{0} \in Y:=\left\{w \in \mathscr{C}^{1}([-1,1]), \quad w( \pm 1)=0\right\} .
$$

It then follows from [Benachour and Dabuleanu 2003, Theorem 3.1 and Proposition 4.1] that the initial-boundary value problem (1)-(3) has a unique classical solution

$$
u \in \mathscr{C}([0, \infty) \times[-1,1]) \cap \mathscr{C}^{2,1}((0, \infty) \times(-1,1))
$$

satisfying

$$
\min _{[-1,1]} u_{0} \leq u(t, x) \leq \max _{[-1,1]} u_{0}, \quad(t, x) \in[0, \infty) \times[-1,1] .
$$

In addition, setting

$$
M(t):=\max _{x \in[-1,1]} u(t, x),
$$

the comparison principle ensures that $t \mapsto M(t)$ is a nonincreasing function of time and we put

$$
M_{\infty}:=\lim _{t \rightarrow \infty} M(t) \in\left[\min _{[-1,1]} u_{0}, \max _{[-1,1]} u_{0}\right] .
$$

We recall that classical solutions to (1)-(3) enjoy the comparison principle; this may be proved by standard arguments, as in [Gilding et al. 2003, Theorem 4]. 
Remark 1. The initial-boundary value problem (1)-(3) is actually well-posed in a larger space than $Y$, which depends on $p$, and we refer to [Benachour and Dabuleanu 2003] for a more detailed account. Still, the solutions constructed in that reference belong to $Y$ for any positive time. Since we are interested here in the large time behaviour, the assumption (5) that $u_{0} \in Y$ is thus not restrictive.

For further use, we also introduce the following notations:

$$
\alpha:=\frac{2-p}{1-p} \quad \text { and } \quad \mu_{0}:=\frac{(1-p)^{\alpha}}{2-p} .
$$

We may now state our main result.

Theorem 2. Consider $u_{0} \in Y$ and denote by $u$ the corresponding classical solution to (1)-(3). Then $M_{\infty} \in\left[0, M_{0}\right]$ and there is a nonnegative stationary solution $u_{s}$ to (1)-(2) such that

$$
\lim _{t \rightarrow \infty}\left\|u(t)-u_{s}\right\|_{\infty}=0 .
$$

Furthermore, $u_{s} \not \equiv 0$ and $M_{\infty}>0$ if

$$
\int_{-1}^{1} u_{0}(x) \cos \left(\frac{\pi x}{2}\right) d x>0 .
$$

It readily follows from the second assertion of Theorem 2 that the set of nontrivial and nonnegative steady states to (1)-(2) attracts all solutions to (1)-(3) starting from a nonnegative initial datum $u_{0} \not \equiv 0$. Observe however that the set of nontrivial and nonnegative steady states to (1)-(2) also attracts sign-changing solutions $u$ to (1)-(3) since there are sign-changing initial data fulfilling (11).

The proof of Theorem 2 requires several steps and is performed as follows: we first identify the stationary solutions to (1)-(2) in Section 2 and use them together with comparison arguments to establish that, if $u_{0} \in Y$ is nonnegative with $u_{0} \not \equiv 0$, then $M_{\infty}>0$ and $\{u(t) ; t \geq 0\}$ is bounded in $\mathscr{C}^{1}([-1,1])$ (Section 3). In Section 4, we employ the technique of [Zelenyak 1968] to construct a Liapunov functional for nonnegative solutions to (1)-(3). Let us mention here that this technique has also been used recently for related problems in [Arrieta et al. 2004; Simondon and Touré 1996]. For nonnegative initial data convergence towards a steady state then follows from the results of Section 3 and Section 4 by a LaSalle invariance principle argument. The large time behaviour of sign-changing initial data is next deduced from that of nonnegative solutions after observing that the negative part of any solution to (1)-(3) vanishes in a finite time (Section 6).

Remark 3. A further outcome of Theorem 2 is that the large behaviour of solutions to (1) on a bounded interval is more complex for homogeneous Dirichlet boundary 
conditions than for periodic and homogeneous Neumann boundary conditions. Indeed, for the latter boundary conditions, it follows from [Benachour and Dabuleanu 2005; Benachour et al. 2002] that there are $T_{\star}>0$ and $m_{\star} \in \mathbb{R}$ such that $u(t) \equiv m_{\star}$ for $t \geq T_{\star}$ whatever the signs of $a$ and $u_{0}$ are.

In Section 7, we prove the extinction in finite time of $\partial_{x} u$ on a subinterval of $(-1,1)$ for a specific class of initial data. More precisely, we have the following result:

Theorem 4. Assume further that there are $m_{0} \in\left(0, M_{0}\right)$ and $\varepsilon>0$ such that

$$
m_{0}-M_{0}|x|^{\alpha}+\varepsilon|x|^{1+\alpha} \leq u_{0}(x) \leq m_{0}, \quad x \in[-1,1] .
$$

Then, for each $t \in(0, \infty)$, there is $X(t) \in(0,1)$ such that

$$
u(t, x)=m_{0} \quad \text { for } \quad x \in(-X(t), X(t)) .
$$

Furthermore, if

$$
\delta_{0}:=1-\left(\frac{m_{0}}{M_{0}}\right)^{1 / \alpha} \in(0,1),
$$

and $\delta \in\left(0, \delta_{0}\right)$, there exists $T(\delta)>0$ such that

$$
u(t, x)=m_{0} \quad \text { for } \quad(t, x) \in[T(\delta), \infty) \times[-\delta, \delta] .
$$

An example of initial datum in $Y$ fulfilling (12) is the following: $u_{0}(x)=M_{0}-$ $\varepsilon-M_{0}|x|^{\alpha}+\varepsilon|x|^{\beta}$ for $x \in[-1,1]$, where $\beta \in(\alpha, \alpha+1]$ and $\varepsilon \in\left(0, \alpha M_{0} / \beta\right)$.

The second assertion of Theorem 4 shows that $\partial_{x} u$ vanishes identically after some time on a subinterval of $[-1,1]$, a phenomenon which one could call finite time incomplete extinction in comparison to what occurs for periodic or homogeneous Neumann boundary conditions. But the first assertion of Theorem 4 reveals that the extinction mechanism is somewhat stronger since, even if $\partial_{x} u_{0}(x)$ vanishes only for $x=0, \partial_{x} u$ vanishes instantaneously on a subinterval of $[-1,1]$ with positive measure.

Another consequence of Theorem 4 and (6) is that $\|u(t)\|_{\infty}=m_{0}$ for every $t \geq 0$. Therefore, for an initial datum $u_{0}$ in $Y$ satisfying (12), the corresponding solution $u$ to (1)-(3) does not obey the strong maximum principle.

The proof of Theorem 4 relies on comparison arguments with travelling wave solutions to (1) and is similar to that of [Gilding 2005, Theorem 9], some care being needed to cope with the boundary conditions. 
Notations. Throughout the paper, we denote by $r_{+}:=\max \{r, 0\}$ the positive part of the real number $r$. For $r \in \mathbb{R}$ and $s \in \mathbb{R}$, we put $r \vee s:=\max \{r, s\}$ and $r \wedge s:=$ $\min \{r, s\}$. Also, for $q \in[1, \infty],\|\cdot\|_{q}$ denotes the $L^{q}(-1,1)$-norm.

\section{Nonnegative steady states}

In this section, we look for nonnegative stationary solutions to (1), (2), that is, nonnegative functions $U \in \mathscr{C}^{2}([-1,1])$ such that

$$
\begin{aligned}
\frac{d^{2} U}{d x^{2}}+\left|\frac{d U}{d x}\right|^{p} & =0, \quad x \in(-1,1), \\
U( \pm 1) & =0 .
\end{aligned}
$$

Proposition 5. Let $U \in \mathscr{C}^{2}([-1,1])$ be a nonnegative solution to (14), (15). Then there is $\vartheta \in[0,1]$ such that $U=U_{\vartheta}$, where

$$
U_{\vartheta}(x):=M_{0}\left[(1-\vartheta)^{\alpha}-(|x|-\vartheta)_{+}^{\alpha}\right], \quad x \in[-1,1] .
$$

Observe that $U_{\vartheta}$ is constant on $[-\vartheta, \vartheta]$ for each $\vartheta \in(0,1)$ and that $U_{1} \equiv 0$. Proof. Let $U \in \mathscr{C}^{2}([-1,1])$ be a nonnegative solution to (14), (15). Then $U$ is concave by (14) and we infer from the nonnegativity of $U$ and the boundary conditions (15) that $d U / d x(-1) \geq 0$ and $d U / d x(1) \leq 0$.

If $d U / d x(-1)=0$, the concavity of $U$ entails that $U$ is a nonincreasing function in $(-1,1)$. Consequently, $U \equiv 0=U_{1}$ to comply with the boundary conditions (15).

Similarly, if $d U / d x(1)=0$, it follows from the concavity of $U$ that $U$ is nondecreasing on $(-1,1)$, whence $U \equiv 0=U_{1}$ by (15).

We finally consider the case where $d U / d x(-1)>0$ and $d U / d x(1)<0$ and put

$$
\begin{aligned}
& x_{I}:=\sup \{X \in(-1,1) \text { such that } d U / d x(x)>0 \text { on }[-1, X)\}, \\
& x_{S}:=\inf \{X \in(-1,1) \text { such that } d U / d x(x)<0 \text { on }(X, 1]\} .
\end{aligned}
$$

Owing to the continuity of $d U / d x$, we have $-1<x_{I} \leq x_{S}<1$ and $d U / d x(x)=0$ for $x \in\left[x_{I}, x_{S}\right]$ by the concavity of $U$. Direct integration of (14) then entails that there are two constants $A$ and $B$ such that

$$
\left|\frac{d U}{d x}(x)\right|^{-p} \frac{d U}{d x}(x)+(1-p) x=\left\{\begin{array}{lll}
A & \text { if } & x \in\left(x_{S}, 1\right], \\
B & \text { if } & x \in\left[-1, x_{I}\right) .
\end{array}\right.
$$

Since $p \in(0,1)$ and $d U / d x$ vanishes for $x \in\left\{x_{I}, x_{S}\right\}$, we may let $x \rightarrow x_{I}$ and $x \rightarrow x_{S}$ in (16) to deduce that $A=(1-p) x_{S}$ and $B=(1-p) x_{I}$. We next integrate (16) to obtain that there are two constants $C_{I}$ and $C_{S}$ such that

$$
U(x)= \begin{cases}C_{S}-M_{0}\left(x-x_{S}\right)^{\alpha} & \text { if } \quad x \in\left(x_{S}, 1\right] \\ C_{I}-M_{0}\left(x_{I}-x\right)^{\alpha} & \text { if } \quad x \in\left[-1, x_{I}\right) .\end{cases}
$$


Requiring the boundary conditions (15) to be fulfilled provides the values of $C_{I}$ and $C_{S}$, whence

$$
U(x)=\left\{\begin{array}{lll}
\mu_{0}\left(1-x_{S}\right)^{\alpha}-\mu_{0}\left(x-x_{S}\right)^{\alpha} & \text { if } \quad x \in\left(x_{S}, 1\right], \\
\mu_{0}\left(x_{I}+1\right)^{\alpha}-\mu_{0}\left(x_{I}-x\right)^{\alpha} & \text { if } \quad x \in\left[-1, x_{I}\right) .
\end{array}\right.
$$

Now, since $d U / d x$ vanishes for $x \in\left[x_{I}, x_{S}\right]$, we shall have $U\left(x_{S}\right)=U\left(x_{I}\right)$, which implies that $1-x_{S}=x_{I}+1$, whence $x_{S}=-x_{I}$. Thus, necessarily, $x_{S} \in[0,1]$, from which the equality $U=U_{x_{S}}$ readily follows.

It is worth mentioning that $\left\|U_{\vartheta}\right\|_{\infty} \leq \mathcal{M}_{0}$ for each $\vartheta \in[0,1]$. Combining this property with the convergence to a steady state to be proved in Section 5, we will conclude that $M_{\infty} \leq M_{0}$.

Remark 6. Proposition 5 shows in particular that there is nonuniqueness of classical solutions to (14), (15). A similar construction is performed in [Alaa and Pierre 1993; Lions 1985] for the boundary-value problem

$$
-\Delta u=|\nabla u|^{p} \text { in } B(0,1), \quad u=0 \text { on } \partial B(0,1),
$$

where $B(0,1)$ denotes the open unit ball of $\mathbb{R}^{N}, N>1$, to establish the nonuniqueness of weak solutions for $p>N /(N-1)$.

\section{Some properties of $\{u(t) ; t \geq 0\}$}

Introducing the positive cone $Y_{+}:=\{w \in Y$ such that $w \geq 0\}$ of $Y$, we first prove that $M_{\infty}>0$ for $u_{0} \in Y_{+}, u_{0} \neq \equiv$, by constructing suitable subsolutions to (1)-(3) with the help of $U_{0}$.

Lemma 7. Let $u_{0} \in Y_{+}$and denote by $u$ the corresponding classical solution to (1)-(3). If $u_{0} \not \equiv 0$, we have $M_{\infty}>0$.

Proof. Since $u_{0} \not \equiv 0$, there are $x_{0} \in(-1,1), \delta \in(0,1)$ and $m>0$ such that $\left(x_{0}-\delta, x_{0}+\delta\right) \subset(-1,1)$ and

$$
u_{0}(x) \geq m \text { for } \quad x \in\left(x_{0}-\delta, x_{0}+\delta\right) .
$$

We put $x_{1}:=\left(x_{0}-1\right) \vee(-1), x_{2}:=\left(x_{0}+1\right) \wedge 1, J:=\left[x_{1}, x_{2}\right]$,

$$
\lambda:=1 \wedge \frac{m}{M_{0}-U_{0}(\delta)},
$$

and $v(x):=\lambda\left(U_{0}\left(x-x_{0}\right)-U_{0}(\delta)\right)$ for $x \in J$.

On the one hand, it follows from (1) and (14) that

$$
\partial_{t} v-\partial_{x}^{2} v-\left|\partial_{x} v\right|^{p}=\left(\lambda-\lambda^{p}\right)\left|\partial_{x} U_{0}\left(.-x_{0}\right)\right|^{p} \leq 0=\partial_{t} u-\partial_{x}^{2} u-\left|\partial_{x} u\right|^{p}
$$


on $[0, \infty) \times J$. On the other hand, the nonnegativity of $u_{0}$ and the maximum principle entail the nonnegativity of $u$ which then warrants that

$$
\begin{aligned}
& v\left(x_{1}\right) \leq v\left(x_{0}-\delta\right)=0 \leq u\left(t, x_{1}\right), \\
& v\left(x_{2}\right) \leq v\left(x_{0}+\delta\right)=0 \leq u\left(t, x_{2}\right),
\end{aligned}
$$

while the choice of $\lambda$ entails that

$$
\begin{aligned}
& v(x) \leq \lambda\left(M_{0}-U_{0}(\delta)\right) \leq m \leq u_{0}(x) \text { for } x \in\left(x_{0}-\delta, x_{0}+\delta\right), \\
& v(x) \leq v\left(x_{0} \pm \delta\right)=0 \leq u_{0}(x) \text { for } x \in J \backslash\left(x_{0}-\delta, x_{0}+\delta\right) .
\end{aligned}
$$

We then infer from the comparison principle that $u(t, x) \geq v(x)$ for $(t, x) \in[0, \infty) \times$ $J$. In particular, $M(t)=\|u(t)\|_{\infty} \geq u\left(t, x_{0}\right) \geq v\left(x_{0}\right)=\lambda\left(M_{0}-U_{0}(\delta)\right)$ for each $t \geq 0$, whence $M_{\infty} \geq \lambda\left(M_{0}-U_{0}(\delta)\right)>0$.

We now turn to the question of global boundedness of the trajectory $\{u(t) ; t \geq 0\}$ in $\mathscr{C}^{1}([-1,1])$.

Lemma 8. Let $u_{0} \in Y_{+}$and denote by $u$ the corresponding classical solution to (1)-(3). There is a constant $\Lambda>0$ depending only on $\left\|u_{0}\right\|_{W^{1, \infty}(-1,1)}$ and $p$ such that

$$
\|u(t)\|_{W^{1, \infty}(-1,1)} \leq \Lambda \text { for } t \geq 0 .
$$

Proof. We first recall that $\{u(t) ; t \geq 0\}$ is bounded in $L^{\infty}(-1,1)$ by $(6)$ and we are left with the proof that $\left\{\partial_{x} u(t) ; t \geq 0\right\}$ is bounded in $L^{\infty}(-1,1)$. For that purpose, we choose $\lambda>1$ such that

$$
\lambda \geq\left[\left(\frac{2}{1-p}\right)^{1 /(1-p)}\left\|\partial_{x} u_{0}\right\|_{\infty}\right] \vee\left[\frac{\left\|u_{0}\right\|_{\infty}}{\left(1-2^{-\alpha}\right) \mu_{0}}\right] .
$$

Putting $v:=\lambda U_{0}$, we first notice that the condition $\lambda>1$ ensures that

$$
\partial_{t} v-\partial_{x}^{2} v-\left|\partial_{x} v\right|^{p}=\left(\lambda-\lambda^{p}\right)\left|\partial_{x} U_{0}\right|^{p} \geq 0 \text { in }(0, \infty) \times(-1,1),
$$

while $v( \pm 1)=u(t, \pm 1)=0$ for each $t \geq 0$. Next, on the one hand, it follows from (19) and the monotonicity properties of $U_{0}$ that, if $x \in(-1 / 2,1 / 2)$, we have

$$
v(x)=\lambda U_{0}(x) \geq \lambda U_{0}(1 / 2)=\lambda \mu_{0}\left(1-2^{-\alpha}\right) \geq\left\|u_{0}\right\|_{\infty} \geq u_{0}(x) .
$$

On the other hand, if $x \in[1 / 2,1]$, we have by (19) that

$$
\begin{aligned}
v(x) & =\lambda\left(U_{0}(x)-U_{0}(1)\right)=\lambda \int_{x}^{1}\left|\frac{d U_{0}}{d x}(y)\right| d y=\alpha \lambda \mu_{0} \int_{x}^{1} y^{1 /(1-p)} d y \\
& \geq \alpha \lambda \mu_{0} \int_{x}^{1} 2^{-1 /(1-p)} d y \geq \int_{x}^{1}\left\|\partial_{x} u_{0}\right\|_{\infty} d y \geq \int_{x}^{1}\left|\partial_{x} u_{0}(y)\right| d y \\
& \geq u_{0}(x) .
\end{aligned}
$$


A similar computation shows that $v(x) \geq u_{0}(x)$ also holds true for $x \in[-1,-1 / 2]$. Therefore, $v \geq u_{0}$ in $[-1,1]$ and the previous analysis allows us to apply the comparison principle and conclude that $u(t, x) \leq v(x)$ for $(t, x) \in[0, \infty) \times[-1,1]$. In particular, if $t \geq 0$ and $x \in(0,1)$, we have

$$
\frac{u(t, x)-u(t, 1)}{x-1}=\frac{u(t, x)}{x-1} \geq \frac{v(x)}{x-1}=\frac{v(x)-v(1)}{x-1} .
$$

Letting $x \rightarrow 1$, we deduce that $\partial_{x} u(t, 1) \geq \partial_{x} v(1)=-\lambda(1-p)^{1 /(1-p)}$. Since $u_{0} \geq 0$, the comparison principle ensures that $u(t, x) \geq 0=u(t, 1)$ for $x \in(0,1)$, so that we also have $\partial_{x} u(t, 1) \leq 0$. Arguing in a similar way for $x=-1$, we end up with

$$
\left|\partial_{x} u(t, \pm 1)\right| \leq \lambda(1-p)^{1 /(1-p)} \text { for } t \geq 0 .
$$

We now put $k:=\left\|\partial_{x} u_{0}\right\|_{\infty} \vee \lambda(1-p)^{1 /(1-p)}, z:=\partial_{x} u$ and $\mathscr{R}:=\{(t, x) \in$ $(0, \infty) \times(-1,1), z(t, x) \neq 0\}$. In the neighbourhood of each point $\left(t_{0}, x_{0}\right)$ of $\mathscr{R}$, the function $\left|\partial_{x} u\right|^{p}$ is smooth, and classical parabolic regularity theory implies that $z$ is $\mathscr{C}^{1,2}$ in a neighbourhood of $\left(t_{0}, x_{0}\right)$ and satisfies

$$
\partial_{t} z(t, x)-\partial_{x}^{2} z(t, x)=p|z(t, x)|^{p-2} z(t, x) \partial_{x} z(t, x) .
$$

Since $\{(t, x) \in(0, \infty) \times(-1,1), z(t, x)>k\} \subset \mathscr{R}$, we deduce from the previous identity and (20) that

$$
\begin{aligned}
\frac{1}{2} \frac{d}{d t}\left\|(z-k)_{+}\right\|_{2}^{2}=\left[(z-k)_{+} \partial_{x} z\right]_{x=-1}^{x=1} & -\int_{-1}^{1}\left|\partial_{x}(z-k)_{+}\right|^{2} d x \\
& +\left[\left(\frac{p}{p+1} z-k\right)|z|^{p} \frac{(z-k)_{+}}{|z-k|}\right]_{x=-1}^{x=1} \\
= & -\int_{-1}^{1}\left|\partial_{x}(z-k)_{+}\right|^{2} d x
\end{aligned}
$$

whence

$$
\left\|(z(t)-k)_{+}\right\|_{2}^{2} \leq\left\|(z(0)-k)_{+}\right\|_{2}^{2}=0,
$$

the last equality being true thanks to the choice of $k$. Consequently, $\partial_{x} u(t, x)=$ $z(t, x) \leq k$ in $[0, \infty) \times[-1,1]$. By a similar argument, we also establish that $\partial_{x} u(t, x)=z(t, x) \geq-k$ in $[0, \infty) \times[-1,1]$. Therefore,

$$
\left|\partial_{x} u(t, x)\right| \leq\left\|\partial_{x} u_{0}\right\|_{\infty} \vee \lambda(1-p)^{1 /(1-p)}
$$

for $(t, x) \in[0, \infty) \times[-1,1]$, which completes the proof of Lemma 8 . 


\section{A Liapunov functional}

We now construct a Liapunov functional for nonnegative solutions to (1)-(3) with the help of the technique developed in [Zelenyak 1968]. Let $u_{0} \in Y_{+}$and denote by $u$ the corresponding classical solution to (1)-(3) which is also nonnegative by the maximum principle. We look for a pair of functions $\Phi$ and $\varrho \geq 0$ such that

$$
\frac{d}{d t} \int_{-1}^{1} \Phi\left(u, \partial_{x} u\right) d x=\int_{-1}^{1} \varrho\left(u, \partial_{x} u\right)\left|\partial_{t} u\right|^{2} d x .
$$

Since $\partial_{t} u(t, \pm 1)=0$ by (2), the first term of the right-hand side of this equality also reads

$$
\begin{aligned}
\frac{d}{d t} \int_{-1}^{1} \Phi & \left(u, \partial_{x} u\right) d x \\
& =\int_{-1}^{1}\left[\partial_{1} \Phi\left(u, \partial_{x} u\right) \partial_{t} u+\partial_{2} \Phi\left(u, \partial_{x} u\right) \partial_{x} \partial_{t} u\right] d x \\
& =\int_{-1}^{1}\left[\partial_{1} \Phi\left(u, \partial_{x} u\right)-\partial_{1} \partial_{2} \Phi\left(u, \partial_{x} u\right) \partial_{x} u-\partial_{2}^{2} \Phi\left(u, \partial_{x} u\right) \partial_{x}^{2} u\right] \partial_{t} u d x
\end{aligned}
$$

and it is then natural to require that

$$
\begin{aligned}
{\left[\partial_{1} \Phi\left(u, \partial_{x} u\right)-\partial_{1} \partial_{2} \Phi\left(u, \partial_{x} u\right) \partial_{x} u-\partial_{2}^{2} \Phi\left(u, \partial_{x} u\right)\right.} & \left.\partial_{x}^{2} u\right] \\
& =\varrho\left(u, \partial_{x} u\right) \partial_{t} u \\
& =\varrho\left(u, \partial_{x} u\right)\left(\left|\partial_{x} u\right|^{p}+\partial_{x}^{2} u\right)
\end{aligned}
$$

for (21) to hold true. Following [Zelenyak 1968], we realize that a sufficient condition for the previous equality to be valid is

$$
\begin{aligned}
\partial_{1} \Phi\left(u, \partial_{x} u\right)-\partial_{1} \partial_{2} \Phi\left(u, \partial_{x} u\right) \partial_{x} u & =\varrho\left(u, \partial_{x} u\right)\left|\partial_{x} u\right|^{p} \\
-\partial_{2}^{2} \Phi\left(u, \partial_{x} u\right) & =\varrho\left(u, \partial_{x} u\right) .
\end{aligned}
$$

Performing the computations as in [Zelenyak 1968], we see that the functions

$$
\Phi\left(u, \partial_{x} u\right):=u-\frac{\left|\partial_{x} u\right|^{2-p}}{(2-p)(1-p)} \quad \text { and } \quad \varrho\left(u, \partial_{x} u\right):=\left|\partial_{x} u\right|^{-p}
$$

solve the differential system (22), (23). However, $\varrho$ is singular when $\partial_{x} u$ vanishes and it is not clear how to give a meaning to (21) for such a choice of functions $\Phi$ and $\varrho$. Nevertherless, we have the following weaker result which turns out to be sufficient for our purposes. 
Proposition 9. For each $t>0$ and $\delta \in(0,1]$, we have

$$
\frac{d}{d t} \int_{-1}^{1}\left(\frac{\left|\partial_{x} u(t, x)\right|^{2-p}}{(2-p)(1-p)}-u(t, x)\right) d x+\int_{-1}^{1} \frac{\left|\partial_{t} u\right|^{2}}{\left(\left|\partial_{x} u\right|^{2}+\delta^{2}\right)^{p / 2}} d x \leq 0
$$

Proof. We fix $\delta \in(0,1]$ and define $\psi_{\varepsilon}$ by

$$
\psi_{\varepsilon}(0)=\psi_{\varepsilon}^{\prime}(0)=0 \quad \text { and } \quad \psi_{\varepsilon}^{\prime \prime}(r)=(|r| \vee \varepsilon)^{-p}, \quad r \in \mathbb{R}
$$

for $\varepsilon \in(0, \delta)$. We infer from (1) and (2) that

$$
\begin{aligned}
\frac{d}{d t} \int_{-1}^{1}\left[\psi_{\varepsilon}\left(\partial_{x} u\right)-u\right] & d x \\
& =\int_{-1}^{1}\left[\psi_{\varepsilon}^{\prime}\left(\partial_{x} u\right) \partial_{x} \partial_{t} u-\partial_{t} u\right] d x \\
& =\left[\psi_{\varepsilon}^{\prime}\left(\partial_{x} u\right) \partial_{t} u\right]_{x=-1}^{x=1}-\int_{-1}^{1}\left[\psi_{\varepsilon}^{\prime \prime}\left(\partial_{x} u\right) \partial_{x}^{2} u+1\right] \partial_{t} u d x \\
& =-\int_{-1}^{1} \psi_{\varepsilon}^{\prime \prime}\left(\partial_{x} u\right)\left(\partial_{x}^{2} u+\left(\left|\partial_{x} u\right| \vee \varepsilon\right)^{p}\right) \partial_{t} u d x \\
& =-\int_{-1}^{1} \psi_{\varepsilon}^{\prime \prime}\left(\partial_{x} u\right)\left(\partial_{t} u+\left(\left|\partial_{x} u\right| \vee \varepsilon\right)^{p}-\left|\partial_{x} u\right|^{p}\right) \partial_{t} u d x \\
& =-\int_{-1}^{1} \psi_{\varepsilon}^{\prime \prime}\left(\partial_{x} u\right)\left|\partial_{t} u\right|^{2} d x-\int_{-1}^{1}\left(1-\frac{\left|\partial_{x} u\right|^{p}}{\varepsilon^{p}}\right)_{+} \partial_{t} u d x
\end{aligned}
$$

On the one hand, since $\varepsilon \in(0, \delta)$, we have

$$
\left|\partial_{x} u\right| \vee \varepsilon \leq\left(\left|\partial_{x} u\right|^{2}+\delta^{2}\right)^{1 / 2},
$$

so that

$$
\int_{-1}^{1} \psi_{\varepsilon}^{\prime \prime}\left(\partial_{x} u\right)\left|\partial_{t} u\right|^{2} d x \geq \int_{-1}^{1} \frac{\left|\partial_{t} u\right|^{2}}{\left(\left|\partial_{x} u\right|^{2}+\delta^{2}\right)^{p / 2}} d x .
$$

On the other hand, introducing

$$
\xi(r):=\left\{\begin{array}{lll}
r-\frac{|r|^{p} r}{(p+1) \varepsilon^{p}} & \text { if } & |r| \leq \varepsilon, \\
\frac{p \varepsilon}{p+1} \frac{r}{|r|} & \text { if } & |r| \geq \varepsilon,
\end{array}\right.
$$


we have $\xi^{\prime}(r)=\left(1-|r|^{p} / \varepsilon^{p}\right)_{+}$and $|\xi(r)| \leq \varepsilon$. Therefore, thanks to (1),

$$
\begin{aligned}
\mid \int_{-1}^{1}\left(1-\frac{\left|\partial_{x} u\right|^{p}}{\varepsilon^{p}}\right) & \partial_{t} u d x \mid \\
& \leq\left|\int_{-1}^{1}\left(1-\frac{\left|\partial_{x} u\right|^{p}}{\varepsilon^{p}}\right)_{+} \partial_{x}^{2} u d x\right|+\varepsilon^{p} \int_{-1}^{1}\left(1-\frac{\left|\partial_{x} u\right|^{p}}{\varepsilon^{p}}\right)_{+} d x \\
\leq & \left|\int_{-1}^{1} \partial_{x} \xi\left(\partial_{x} u\right) d x\right|+2 \varepsilon^{p} \\
\leq & \left|\xi\left(\partial_{x} u(t, 1)\right)\right|+\left|\xi\left(\partial_{x} u(t,-1)\right)\right|+2 \varepsilon^{p} \leq 4 \varepsilon^{p} .
\end{aligned}
$$

Consequently, for each $\varepsilon \in(0, \delta)$, we have

$$
\frac{d}{d t} \int_{-1}^{1}\left[\psi_{\varepsilon}\left(\partial_{x} u\right)-u\right] d x+\int_{-1}^{1} \frac{\left|\partial_{t} u\right|^{2}}{\left(\left|\partial_{x} u\right|^{2}+\delta^{2}\right)^{p / 2}} d x \leq 4 \varepsilon^{p}
$$

It remains to pass to the limit in $(25)$ as $\varepsilon \rightarrow 0$. For that purpose, we notice that

$$
\left|\psi_{\varepsilon}^{\prime}(r)-\frac{|r|^{-p} r}{1-p}\right| \leq \frac{p}{1-p} \varepsilon^{1-p}
$$

for $r \in \mathbb{R}$, so that $\left(\psi_{\varepsilon}\right)$ converges uniformly towards $r \mapsto|r|^{2-p} /((2-p)(1-p))$ on compact subsets of $\mathbb{R}$. Recalling that $\partial_{x} u(t)$ belongs to $L^{\infty}(-1,1)$ by Lemma 8 , we may let $\varepsilon \rightarrow 0$ in (25) and obtain (24).

Remark 10. It turns out that, at least formally, the functional

$$
w \mapsto \int_{-1}^{1}\left(\frac{\left|\partial_{x} w(x)\right|^{2-p}}{(2-p)(1-p)}-w(x)\right) d x
$$

is also a Liapunov functional for (1)-(3) when $p \in(1,2)$, while

$$
w \mapsto \int_{-1}^{1}\left(\left|\partial_{x} w(x)\right| \ln \left(\left|\partial_{x} w(x)\right|\right)-\left|\partial_{x} w(x)\right|-w(x)\right) d x
$$

is a Liapunov functional for (1)-(3) when $p=1$. For $p>2$, (1)-(3) still have Liapunov functionals but of a different kind [Arrieta et al. 2004].

Corollary 11. We have

$$
\int_{0}^{\infty} \int_{-1}^{1}\left|\partial_{t} u(t, x)\right|^{2} d x d t<\infty
$$


Proof. Let $T>0$. We integrate (24) with $\delta=1$ over $(0, T)$ and use (18) and the nonnegativity of $u$ to obtain

$$
\begin{aligned}
\int_{0}^{T} & \int_{-1}^{1} \frac{\left|\partial_{t} u(t, x)\right|^{2}}{\left(1+\Lambda^{2}\right)^{p / 2}} d x d t \\
& \leq \int_{0}^{T} \int_{-1}^{1} \frac{\left|\partial_{t} u(t, x)\right|^{2}}{\left(\left|\partial_{x} u(t, x)\right|^{2}+1\right)^{p / 2}} d x d t \\
& \leq \int_{-1}^{1}\left(\frac{\left|\partial_{x} u(0, x)\right|^{2-p}}{(2-p)(1-p)}-u(0, x)\right) d x-\int_{-1}^{1}\left(\frac{\left|\partial_{x} u(T, x)\right|^{2-p}}{(2-p)(1-p)}-u(T, x)\right) d x \\
& \leq \frac{2\left\|\partial_{x} u_{0}\right\|_{\infty}^{2-p}}{(2-p)(1-p)}+\int_{-1}^{1} u(T, x) d x \leq \frac{2\left\|\partial_{x} u_{0}\right\|_{\infty}^{2-p}}{(2-p)(1-p)}+2 \Lambda .
\end{aligned}
$$

Since the right-hand side does not depend on $T>0$, we deduce (26).

\section{Convergence to steady states}

Proof of Theorem 2: nonnegative initial data. Let $u_{0} \in Y_{+}, u_{0} \neq \equiv 0$, and denote by $u$ the corresponding classical solution to (1)-(3). We consider an increasing sequence $\left(t_{n}\right)_{n \geq 1}$ of positive real numbers such that $t_{n} \rightarrow \infty$ as $n \rightarrow \infty$ and define a sequence of functions $\left(u_{n}\right)_{n \geq 1}$ by $u_{n}(t, x):=u\left(t_{n}+t, x\right)$ for $(t, x) \in[0,1] \times[-1,1]$ and $n \geq 1$. We next denote by $g_{n}$ the solution to

$$
\begin{aligned}
\partial_{t} g_{n}-\partial_{x}^{2} g_{n} & =0, \quad(t, x) \in(0,1) \times(-1,1), \\
g_{n}(t, \pm 1) & =0, \quad t \in(0,1), \\
g_{n}(0) & =u_{n}(0)=u\left(t_{n}\right), \quad x \in(-1,1),
\end{aligned}
$$

and put $h_{n}=u_{n}-g_{n}$. Then $h_{n}$ is a solution to

$$
\begin{aligned}
\partial_{t} h_{n}-\partial_{x}^{2} h_{n} & =\left|\partial_{x} u_{n}\right|^{p}, \quad(t, x) \in(0,1) \times(-1,1), \\
h_{n}(t, \pm 1) & =0, \quad t \in(0,1), \\
h_{n}(0) & =0, \quad x \in(-1,1) .
\end{aligned}
$$

By Lemma 8 , the sequence $\left(\left|\partial_{x} u_{n}\right|^{p}\right)$ is bounded in $L^{q}((0,1) \times(-1,1))$ for every $q \in(1, \infty)$. Since $h_{n}$ is a solution to (30)-(32), we infer from [Ladyženskaja et al. 1968, Theorem IV.9.1] that $\left(h_{n}\right)$ is bounded in $\left\{w \in L^{q}\left(0,1 ; W^{2, q}(-1,1)\right), \partial_{t} w \in\right.$ $\left.L^{q}((0,1) \times(-1,1))\right\}$ for every $q \in(1, \infty)$. We may then use [Ladyženskaja et al. 1968, Lemma II.3.3] with $q=4$ to deduce that there is $\beta \in(0,1)$ such that $\left(h_{n}\right)$ and $\left(\partial_{x} h_{n}\right)$ are bounded in $\mathscr{C}^{\beta / 2, \beta}([0,1] \times[-1,1])$. This last property together with the Arzelà-Ascoli theorem entail that $\left(h_{n}\right)$ and $\left(\partial_{x} h_{n}\right)$ are relatively compact in $\mathscr{C}([0,1] \times[-1,1])$. 
At the same time, it follows from Lemma 8 and classical regularity properties of the heat equation that $\left(g_{n}\right)$ is relatively compact in $\mathscr{b}([0,1] \times[-1,1])$, while $\left(\partial_{x} g_{n}\right)$ is relatively compact in $\mathscr{C}([\tau, 1] \times[-1,1])$ for each $\tau \in(0,1)$. Consequently, there are a subsequence of $\left(u_{n}\right)$ (not relabeled) and $U \in \mathscr{C}([0,1] \times[-1,1])$ such that $\partial_{x} U \in \mathscr{C}((0,1] \times[-1,1])$ and

$$
\begin{array}{ccc}
u_{n} & \longrightarrow U & \text { in } \mathscr{C}([0,1] \times[-1,1]), \\
\partial_{x} u_{n} & \longrightarrow \partial_{x} U & \text { in } \mathscr{C}([\tau, 1] \times[-1,1])
\end{array}
$$

for every $\tau \in(0,1)$.

Now, since $\left(u_{n}\right)$ satisfies (1), (2), a straightforward consequence of (33) is that

$$
\partial_{t} U-\partial_{x}^{2} U=\left|\partial_{x} U\right|^{p} \text { in } \mathscr{D}^{\prime}((0,1) \times(-1,1)) .
$$

Furthermore, it follows from Corollary 11 that

$$
\lim _{n \rightarrow \infty} \int_{0}^{1} \int_{-1}^{1}\left|\partial_{t} u_{n}\right|^{2} d x d t=\lim _{n \rightarrow \infty} \int_{t_{n}}^{1+t_{n}} \int_{-1}^{1}\left|\partial_{t} u\right|^{2} d x d t=0 .
$$

By a weak lower semicontinuity argument, we infer from (33) and the previous identity that $\partial_{t} U=0$. Then $U$ does not depend on time and thus belongs to $\mathscr{C}^{1}([-1,1])$. Furthermore, recalling (34), we conclude that $\partial_{x}^{2} U+\left|\partial_{x} U\right|^{p}=0$ in $\mathscr{D}^{\prime}(-1,1)$. The already established regularity of $U$ implies that $U \in \mathscr{C}^{2}([-1,1])$ and solves (14), (15). Consequently, by Proposition 5, there exists $\vartheta \in[0,1]$ such that $U=U_{\vartheta}$ and $\left(u_{n}(0)\right)=\left(u\left(t_{n}\right)\right)$ converges towards $U_{\vartheta}$ in $\mathscr{C}([-1,1])$ as $n \rightarrow \infty$ by (33). In particular, recalling that $M(t)$ is defined by (7), we have

$$
M_{0}(1-\vartheta)^{\alpha}=\left\|U_{\vartheta}\right\|_{\infty}=\lim _{n \rightarrow \infty}\left\|u\left(t_{n}\right)\right\|_{\infty}=\lim _{n \rightarrow \infty} M\left(t_{n}\right)=M_{\infty},
$$

whence $M_{\infty} \leq M_{0}$ and

$$
\vartheta=1-\left(\frac{M_{\infty}}{M_{0}}\right)^{1 / \alpha}
$$

Since this identity determines $\vartheta$ in a unique way, we deduce that the set of cluster points of $\{u(t) ; t \geq 0\}$ is reduced to a single point $\left\{U_{\vartheta}\right\}$ with $\vartheta$ given by (35). The set $\{u(t) ; t \geq 0\}$ being relatively compact in $\mathscr{C}([-1,1])$ by Lemma 8 and the Arzelà-Ascoli theorem, we finally conclude that $\left\|u(t)-U_{\vartheta}\right\|_{\infty} \rightarrow 0$ as $t \rightarrow \infty$, whence (10). In addition, since $u_{0} \not \equiv 0$, Lemma 7 guarantees that $\vartheta<1$, so that $U_{\vartheta}$ is indeed a nontrivial steady state to (1)-(3). We have thus proved that,

$$
\begin{aligned}
& \text { if } u_{0} \in Y_{+}, u_{0} \not \equiv 0 \text {, then } M_{\infty}>0 \text { and there is } \vartheta \in[0,1) \text { such that } \\
& \left\|u(t)-U_{\vartheta}\right\|_{\infty} \rightarrow 0 \text { as } t \rightarrow \infty,
\end{aligned}
$$

and Theorem 2 holds true for nonnegative initial data. 


\section{Sign-changing solutions}

We now show that the family $\left(U_{\vartheta}\right)_{\vartheta \in[0,1]}$ of nonnegative steady states to (1)(2) constructed in Proposition 5 also describes the large time behaviour of signchanging solutions to (1)-(3). For that purpose, we first establish that any solution to (1)-(3) becomes nonnegative after a finite time.

Lemma 12. Consider $u_{0} \in Y$ and denote by $u$ the corresponding classical solution to (1)-(3). Then there is $T_{\star}>0$ such that $u(t, x) \geq 0$ for $(t, x) \in\left[T_{\star}, \infty\right) \times[-1,1]$. Moreover, if $u_{0} \leq 0$, then $u(t, x)=0$ for $(t, x) \in\left[T_{\star}, \infty\right) \times[-1,1]$.

Proof. We put $\tilde{u}_{0}(x)=0 \wedge u_{0}(x)$ for $x \in[-1,1]$ and $\tilde{u}_{0}(x)=0$ for $x \in \mathbb{R} \backslash$ $[-1,1]$. Since $\tilde{u}_{0}$ is a nonpositive, bounded and continuous function in $\mathbb{R}$, we infer from [Gilding et al. 2003, Theorem 3] that there is a unique classical solution $\left.\tilde{u} \in \mathscr{C}([0, \infty) \times \mathbb{R}) \cap \mathscr{C}^{1,2}((0, \infty) \times \mathbb{R})\right)$ to the Cauchy problem

$$
\begin{aligned}
\partial_{t} \tilde{u}-\partial_{x}^{2} \tilde{u} & =a\left|\partial_{x} \tilde{u}\right|^{p}, \quad(t, x) \in(0, \infty) \times \mathbb{R}, \\
\tilde{u}(0) & =\tilde{u}_{0}, \quad x \in \mathbb{R} .
\end{aligned}
$$

Furthermore, $\tilde{u}$ is nonpositive in $(0, \infty) \times \mathbb{R}$ and is thus clearly a subsolution to (1)-(3) since $\tilde{u}_{0} \leq u_{0}$. The comparison principle then entails that

$$
\tilde{u}(t, x) \leq u(t, x) \text { for }(t, x) \in[0, \infty) \times[-1,1] .
$$

But, since $\tilde{u}_{0}$ is a nonpositive, bounded and continuous function with compact support in $\mathbb{R}$, it follows from [Benachour et al. 2002; Gilding 2005] that $\tilde{u}$ enjoys the property of finite time extinction, that is, there is $T_{\star}>0$ such that

$$
\tilde{u}(t, x)=0 \text { for }(t, x) \in\left[T_{\star}, \infty\right) \times \mathbb{R} .
$$

Combining these two facts yield the first assertion of Lemma 12. Next, if $u_{0} \leq 0$, we have also $u \leq 0$ in $[0, \infty) \times[-1,1]$ by (6) and $u$ thus identically vanishes in $\left[T_{\star}, \infty\right) \times[-1,1]$.

Proof of Theorem 2: sign-changing initial data. By Lemma 12, there is $T_{\star}>0$ such that $u\left(T_{\star}, x\right) \geq 0$ for $x \in[-1,1]$. Then either $u\left(T_{\star}\right) \equiv 0$ and thus $u(t) \equiv 0$ for $t \geq T_{\star}$, and $u(t)$ converges towards $U_{1}$ as $t \rightarrow \infty$. Or $u\left(T_{\star}\right) \not \equiv 0$ and we infer from (36) that there is $\vartheta \in[0,1)$ such that $u\left(t+T_{\star}\right)$ converges towards $U_{\vartheta}$ as $t \rightarrow \infty$, which completes the proof of the first statement of Theorem 2.

Assume next that $u_{0}$ fulfils (11). Putting $\varphi_{1}(x):=\cos (\pi x / 2)$ for $x \in[-1,1]$ and $\lambda_{1}:=\pi^{2} / 4$, we recall that $-d^{2} \varphi_{1} / d x^{2}=\lambda_{1} \varphi_{1}$ in $(-1,1)$ with $\varphi_{1}( \pm 1)=0$. We infer from (1), (11) and the nonnegativity of $\varphi_{1}$ and $\left|\partial_{x} u\right|^{p}$ that

$$
\int_{-1}^{1} u(t, x) \varphi_{1}(x) d x \geq e^{-\lambda_{1} t} \int_{-1}^{1} u_{0}(x) \varphi_{1}(x) d x>0
$$


for $t \geq 0$. In particular, with the previous notations, we have $u\left(T_{\star}\right) \geq 0$ with

$$
\int_{-1}^{1} u\left(T_{\star}, x\right) \varphi_{1}(x) d x>0,
$$

which, together with the positivity of $\varphi_{1}$ on $(-1,1)$, ensures that $u\left(T_{\star}\right)$ is nonnegative with $u\left(T_{\star}\right) \not \equiv 0$. Arguing as before, we infer from (36) that there is $\vartheta \in[0,1)$ such that $u(t)$ converges towards $U_{\vartheta}$ as $t \rightarrow \infty$, which completes the proof of the second statement of Theorem 2.

\section{Partial extinction of $\partial_{x} u$ in finite time}

Before proceeding with the proof of Theorem 4, we recall that, if $\sigma \in(0, \infty)$ and $\mu \in \mathbb{R}$, the function $(t, x) \mapsto \mu+W_{\sigma}(x-\sigma t)$ is a travelling wave solution to $\partial_{t} w-\partial_{x}^{2} w=\left|\partial_{x} w\right|^{p}$ in $(0, \infty) \times \mathbb{R}$ (see [Gilding and Kersner 2004, Chapter 13], for instance), where

$$
W_{\sigma}(\xi):=-\sigma^{-1 /(1-p)} \int_{0}^{\xi}\left(1-e^{-\sigma(1-p) \eta}\right)_{+}^{1 /(1-p)} d \eta, \quad \xi \in \mathbb{R} .
$$

Introducing $W_{0}(\xi)=-M_{0} \xi_{+}^{\alpha}$ for $\xi \in \mathbb{R}$, we claim that

$$
0 \leq W_{\sigma}(\xi)-W_{0}(\xi) \leq \sigma \kappa_{p} \xi_{+}^{1+\alpha}, \quad \xi \in \mathbb{R},
$$

with $\kappa_{p}:=(1-p)^{\alpha} /(2(3-2 p))$. Indeed, introducing $\zeta(r):=\left(r-1+e^{-r}\right) / r^{2}$ and $\zeta_{1}(r):=r \zeta(r)$ for $r \geq 0$, we have for $\xi \geq 0$

$$
W_{\sigma}(\xi)-W_{0}(\xi)=\int_{0}^{\xi}((1-p) \eta)^{1 /(1-p)}\left\{1-\left(1-\zeta_{1}(\sigma(1-p) \eta)\right)^{1 /(1-p)}\right\} d \eta .
$$

We deduce from the elementary inequalities $0 \leq \zeta_{1}(r) \leq 1$ for $r \geq 0$ and

$$
(1-r)^{1 /(1-p)} \geq 1-\frac{r}{1-p}, \quad r \in[0,1],
$$

that $W_{\sigma}(\xi)-W_{0}(\xi) \geq 0$ and

$$
W_{\sigma}(\xi)-W_{0}(\xi) \leq \int_{0}^{\xi}((1-p) \eta)^{1 /(1-p)} \frac{\zeta_{1}(\sigma(1-p) \eta)}{1-p} d \eta .
$$

We next use the fact that $\zeta(r) \leq 1 / 2$ for $r \geq 0$ to complete the proof of (40).

Proof of Theorem 4. As mentioned, the proof is similar to that of [Gilding 2005, Theorem 9], the main difference being due to the boundary conditions. We nevertheless reproduce the whole argument here for the sake of completeness. We first observe that (12) implies that $u_{0}(x) \geq m_{0}-M_{0}+U_{0}(x)$ for $x \in[-1,1]$ and that 
$m_{0}-M_{0}+U_{0}$ is a subsolution to (1) with $m_{0}-M_{0}+U_{0}( \pm 1) \leq 0$. We then infer from the comparison principle and (6) that

$$
m_{0}-M_{0}+U_{0}(x) \leq u(t, x) \leq m_{0} \text { for }(t, x) \in[0, \infty) \times[-1,1] .
$$

In particular,

$$
u(t, 0)=m_{0} \text { for } t \in[0, \infty) .
$$

We now consider $\sigma \in\left(0, \varepsilon / \kappa_{p}\right)$ and put $w_{\sigma}(t, x)=m_{0}+W_{\sigma}(x-\sigma t)$ for $(t, x) \in$ $[0, \infty) \times \mathbb{R}$ (recall that $\varepsilon$ and $m_{0}$ are both defined in (12)). We readily have that

$$
\partial_{t} w_{\sigma}-\partial_{x}^{2} w_{\sigma}-\left|\partial_{x} w_{\sigma}\right|^{p}=0=\partial_{t} u-\partial_{x}^{2} u-\left|\partial_{x} u\right|^{p} \quad \text { in } \quad(0, \infty) \times(0,1)
$$

with

$$
w_{\sigma}(t, 0)=m_{0}=u(t, 0), \quad t \geq 0,
$$

by (39) and (42). In addition, we infer from (12), (40) and the choice of $\sigma$ that, for $x \in[0,1]$,

$$
\begin{aligned}
w_{\sigma}(0, x) & =m_{0}+W_{\sigma}(x)=m_{0}+W_{0}(x)+W_{\sigma}(x)-W_{0}(x) \\
& \leq m_{0}-M_{0} x^{\alpha}+\sigma \kappa_{p} x^{1+\alpha} \leq m_{0}-M_{0} x^{\alpha}+\varepsilon x^{1+\alpha} \\
& \leq u_{0}(x) .
\end{aligned}
$$

Finally, if $\delta \in\left(0, \delta_{0}\right)$ and $t \in[0, \delta / \sigma]$, it follows from (40) that

$$
\begin{aligned}
w_{\sigma}(t, 1) & =m_{0}+W_{\sigma}(1-\sigma t) \\
& =m_{0}+W_{0}(1-\sigma t)+W_{\sigma}(1-\sigma t)-W_{0}(1-\sigma t) \\
& \leq m_{0}-M_{0}(1-\sigma t)^{\alpha}+\sigma \kappa_{p}(1-\sigma t)^{1+\alpha} \\
& \leq M_{0}\left(\left(1-\delta_{0}\right)^{\alpha}-(1-\delta)^{\alpha}\right)+\sigma \kappa_{p} \\
& \leq 0
\end{aligned}
$$

as soon as $\sigma$ is sufficiently small. Owing to (43), (44), (45) and (46), there is $\sigma_{\delta}$ depending only on $p, m_{0}, \varepsilon$ and $\delta$ such that, if $\sigma \in\left(0, \sigma_{\delta}\right)$, we may apply the comparison principle on $[0, \delta / \sigma] \times[0,1]$ to deduce that

$$
w_{\sigma}(t, x) \leq u(t, x), \quad(t, x) \in[0, \delta / \sigma] \times[0,1] .
$$

Recalling (41), we conclude from (47) that, if $\sigma \in\left(0, \sigma_{\delta}\right)$,

$$
u(t, x)=m_{0} \text { for } t \in[0, \delta / \sigma] \text { and } x \in[0, \sigma t] .
$$

A first consequence of (47) is that, if $t>0$, we may find $\sigma$ small enough such that $\sigma \in\left(0, \sigma_{\delta}\right)$ and $t \in[0, \delta / \sigma]$. It then follows from (48) that $u(t, x)=m_{0}$ for $x \in[0, X(t)]$ with $X(t):=\sigma t$. 
As a second consequence of (47), we note that, if $t \geq T(\delta):=\delta / \sigma_{\delta}$, there is $\sigma \in\left(0, \sigma_{\delta}\right)$ such that $t=\delta / \sigma$. Then $u(t, x)=m_{0}$ for $x \in[0, \delta]$ by (48).

To complete the proof of Theorem 4, it suffices to notice that $v:(t, x) \mapsto$ $u(t,-x)$ also solves (1)-(2) with initial datum $x \mapsto u_{0}(-x)$ which satisfies (12). Then, $v$ also enjoys the above two properties from which we deduce that we have also $u(t, x)=m_{0}$ for $x \in[-X(t), 0]$ for every $t>0$ and $u(t, x)=m_{0}$ for $x \in[-\delta, 0]$ and $t \geq T(\delta)$, thus completing the proof of Theorem 4 .

\section{Acknowledgements}

Part of this work was done while the author enjoyed the hospitality and support of the Helsinki University of Technology and the University of Helsinki, within the Finnish Mathematical Society Visitor Program in Mathematics 2005-2006 on Function Spaces and Differential Equations. I also thank Saïd Benachour, Brian Gilding, Michel Pierre and Philippe Souplet for helpful discussions and comments, and the referee for pertinent remarks.

\section{References}

[Alaa and Pierre 1993] N. E. Alaa and M. Pierre, "Weak solutions of some quasilinear elliptic equations with data measures”, SIAM J. Math. Anal. 24 (1993), 23-35. MR 94g:35091 Zbl 0809.35021

[Arrieta et al. 2004] J. M. Arrieta, A. Rodriguez-Bernal, and P. Souplet, "Boundedness of global solutions for nonlinear parabolic equations involving gradient blow-up phenomena", Ann. Sc. Norm. Super. Pisa Cl. Sci. (5) 3:1 (2004), 1-15. MR 2005b:35122 Zbl 1072.35098

[Benachour and Dabuleanu 2003] S. Benachour and S. Dabuleanu, "The mixed Cauchy-Dirichlet problem for a viscous Hamilton-Jacobi equation”, Adv. Differential Equations 8:12 (2003), 14091452. MR 2005e:35108 Zbl 1101.35043

[Benachour and Dabuleanu 2005] S. Benachour and S. Dabuleanu, "Large time behavior for a viscous Hamilton-Jacobi equation with Neumann boundary condition", J. Differential Equations 216:1 (2005), 223-258. MR 2006d:35104 Zbl 1078.35055

[Benachour et al. 2002] S. Benachour, P. Laurençot, D. Schmitt, and P. Souplet, "Extinction and nonextinction for viscous Hamilton-Jacobi equations in $\mathbb{R}^{N}$ ", Asymptot. Anal. 31:3-4 (2002), 229-246. MR 2003i:35125 Zbl 1046.35053

[Benachour et al. 2007] S. Benachour, S. Dăbuleanu-Hapca, and P. Laurençot, "Decay estimates for a viscous Hamilton-Jacobi equation with homogeneous Dirichlet boundary conditions", Asymptot. Anal. 51:3-4 (2007), 209-229.

[Gilding 2005] B. H. Gilding, "The Cauchy problem for $u_{t}=\Delta u+|\nabla u|^{q}$, large-time behaviour", J. Math. Pures Appl. (9) 84:6 (2005), 753-785. MR 2006a:35149 Zbl 02244568

[Gilding and Kersner 2004] B. H. Gilding and R. Kersner, Travelling waves in nonlinear diffusionconvection reaction, Prog. Nonlinear Diff. Eq. Appl. 60, Birkhäuser, Basel, 2004. MR 2005k:35002 Zbl 1073.35002

[Gilding et al. 2003] B. H. Gilding, M. Guedda, and R. Kersner, "The Cauchy problem for $u_{t}=$ $\Delta u+|\nabla u|^{q} ”$, J. Math. Anal. Appl. 284:2 (2003), 733-755. MR 2005h:35165 Zbl 1041.35026 
[Ladyženskaja et al. 1968] O. A. Ladyženskaja, V. A. Solonnikov, and N. N. Ural'ceva, Linear and quasi-linear equations of parabolic type, Transl. Math. Monogr. 23, Amer. Math. Soc., Providence, RI, 1968. MR 39 \#3159a Zbl 0174.15403

[Lions 1985] P.-L. Lions, "Quelques remarques sur les problèmes elliptiques quasilinéaires du second ordre", J. Analyse Math. 45 (1985), 234-254. MR 87f:35088 Zbl 0614.35034

[Simondon and Touré 1996] F. Simondon and H. Touré, "A Lyapunov functional and long-time behaviour for a degenerate parabolic problem", Adv. Math. Sci. Appl. 6:1 (1996), 243-266. MR 97a:35129 Zbl 0860.35064

[Souplet 2002] P. Souplet, "Gradient blow-up for multidimensional nonlinear parabolic equations with general boundary conditions”, Diff. Integral Eq. 15:2 (2002), 237-256. MR 2002h:35128 Zbl 1015.35016

[Souplet and Zhang 2006] P. Souplet and Q. S. Zhang, "Global solutions of inhomogeneous Hamilton-Jacobi equations”, J. Analyse Math. 99 (2006), 355-396. MR 2279557

[Zelenyak 1968] T. I. Zelenyak, "Stabilization of solutions of boundary value problems for a second order parabolic equation with one space variable", 4 (1968), 34-45. In Russian; translated in Differential Equations 4 (1968), 17-22. MR 36 \#6806 Zbl 0162.15301

Received October 24, 2005.

\section{PHILIPPE LAURENÇOT}

MATHÉMATIQUes POUR L'INDUSTRIE ET LA PhySiQue, CNRS UMR 5640

UNIVERSité PAUl SABATIER - TOULOUSE 3

118 ROUTE DE NARBONNE

F-31062 TOULOUSE CEDEX 9

FRANCE

laurenco@mip.ups-tlse.fr

http://www.mip.ups-tlse.fr/ laurenco/ 